\title{
Gas-phase recombination, grain neutralization and cosmic-ray ionization in diffuse gas
}

\author{
H. Liszt ${ }^{\star}$ \\ National Radio Astronomy Observatory, 520 Edgemont Road, Charlottesville, VA 22903-2475, USA \\ Received 17 September 2002 / Accepted 12 November 2002

\begin{abstract}
Atomic ions are mostly neutralized by small grains (or PAH molecules) in current theories of heating and cooling in cool diffuse clouds; in the main they do not recombine with free electrons. This alters the ionization balance by depressing $n\left(\mathrm{H}^{+}\right)$ and $n\left(\mathrm{He}^{+}\right)$while carbon generally remains nearly fully once-ionized: charge exchange with atomic oxygen and formation of $\mathrm{H}_{2}$ and $\mathrm{OH}$ also depress $n\left(\mathrm{H}^{+}\right)$in partly molecular gas. Seemingly restrictive empirical limits on $\zeta_{\mathrm{H}}$ are relaxed and higher values for $\zeta_{\mathrm{H}}$ are favored in a wide range of circumstances, when grain neutralization is recognized. Maintenance of the proton density at levels needed to reproduce observations of HD requires $\zeta_{\mathrm{H}} \gtrsim 2 \times 10^{-16} \mathrm{~s}^{-1}$, but such models naturally explain the presence of both $\mathrm{HD}$ and $\mathrm{H}_{3}^{+}$in relatively tenuous $\mathrm{H}$ I clouds. In dense gas, a higher ionization rate can account for high observed fractions of atomic hydrogen, and recognition of the effects of grain neutralization can resolve a major paradox in the formation of sulfur-bearing compounds.
\end{abstract}

Key words. ISM: general - ISM: cosmic rays - ISM: atoms - ISM: molecules

\section{Introduction}

Several authors (Wolfire et al. 1995; Liszt 2001; Weingartner \& Draine 2001b; Welty \& Hobbs 2001) have commented recently on a seeming side-effect of current theories in which the heating of the diffuse ISM is dominated by the photoelectric effect on small grains or PAH molecules (Omont 1986; D'Hendecourt \& Leger 1987; Lepp \& Dalgarno 1988b; Bakes \& Tielens 1994; Weingartner \& Draine 2001c). The usual processes of direct radiative and dielectronic recombination of atomic ions become secondary to neutralization during gas-grain interactions. When gas is somewhat denser or shielded from the interstellar diffuse soft X-ray flux, the proton density is much lower than would otherwise be the case and the total ionization fraction is much more nearly that given by the amount of free carbon in the gas alone; carbon remains mostly once ionized.

This neutralization process was discussed by Draine \& Sutin (1987), Lepp \& Dalgarno (1988a) and Lepp et al. (1988) and is not limited to diffuse gas or hydrogen ions. Indeed, ion stage ratios like $\mathrm{Mg}$ II/Mg I, C II/C I etc. should be entirely controlled in this way if large molecules cum small grains heat the neutral gas. All this notwithstanding, recognition of the importance of grain neutralization has occurred only with great hesitation, observational demonstrations of its relevance are still largely lacking and it is typically noted by observers (if at all) as something of a curiosity: Welty \& Hobbs (2001) is an obvious exception. The original authors were concerned with establishing the existence of the effect and determining the abundance

\footnotetext{
* e-mail: hliszt@nrao.edu
}

of the responsible agents, postulating what they described as ad hoc modifications of the chemistry. Now, with the benefit of hindsight and with general acceptance of the heating effect of the same population of small grains, it is appropriate to recognize that the higher ion-neutralization rates associated with gasgrain interaction have other, largely unexplored consequences for our understanding of the interstellar medium (ISM).

The approach taken here to studying these effects is largely based on observations of various forms of hydrogen because they are uniquely sensitive to the cosmic-ray ionization rate in gas shielded from the diffuse soft X-ray flux. Determination of the proton density, cosmic-ray ionization rate and efficacy of grain neutralization are inextricably intertwined. We show that some important historical limits on $\zeta_{\mathrm{H}}$ (the cosmic-ray ionization rate of hydrogen, defined as the primary rate per neutral atom) must be restated, because they were derived only in terms of explicit ratios $\zeta_{\mathrm{H}} / \alpha_{\text {gas }}$, where (symbolically) $\alpha_{\text {gas }}$, the rate for gas-phase recombination with an electron, grossly underestimates the actual rate at which ions are neutralized. Relaxation of these limits allows recognition that essentially all observations of the various forms of hydrogen (protons, $\mathrm{HD}, \mathrm{H}_{2}$ and $\mathrm{H}_{3}^{+}$in more diffuse gas; $\mathrm{H} \mathrm{I}$ in dark molecular gas) suggest a much-enhanced cosmic-ray ionization rate.

The combination of faster neutralization of atomic ions which carry the charge in diffuse gas - and faster cosmic ray ionization has strong implications for the chemistry of diffuse, partly molecular gas. Unlike atoms, molecular ions are dissociated by recombination more rapidly than they are neutralized by grains. If the cosmic-ray flux is enhanced, producing 
molecular ions more rapidly, without a concommitant increase in either the electron fraction or the molecular ion destruction rate, the abundances of molecular ions may be much enhanced: $\mathrm{HD}$ and $\mathrm{H}_{3}^{+}$may coexist in the observed amounts, even in gas of fairly low density.

In the next section, we lay out some of the underlying ionization and ion neutralization mechanisms in the context of recent two-phase heating/cooling models of the ISM. In Sect. 3 we discuss some received limits on the proton density and cosmic ray ionization rate in atomic gas, and demonstrate that limits on $\zeta_{\mathrm{H}}$ from radio recombination lines are not restrictive if grain neutralization occurs; we conclude that ionizing fluxes could be much higher than previously assumed. In Sect. 4 we explore some consequences of the combination of grain neutralization and higher $\zeta_{\mathrm{H}}$ for the chemistry of diffuse clouds and we show that these include a much-enhanced abundance of $\mathrm{H}_{3}^{+}$, as has recently been seen in the ISM (McCall et al. 2002; Geballe et al. 1999), in the same gas which harbors HD. Section 5 is a brief discussion of processes in dark, fully molecular gas.

\section{Physical models of ionization, recombination and the ISM}

\subsection{Ionization in two-phase models of heating and cooling in the ISM}

Our discussion is based on a calculation of heating and cooling very similar to that set out by Wolfire et al. (1995); a spirited defense of classical notions of two-phase equilibrium, in the presence of other influences, has recently been given by Wolfire et al. (2003). This model has been used by us to discuss $\mathrm{H}_{2}$ and CO formation in diffuse clouds (Liszt \& Lucas 2000), the spin temperature of $\mathrm{H}$ I in warm gas (it is not thermalized by collisions, see Liszt 2001) and the formation of $\mathrm{H}_{2}$ in damped Lyman- $\alpha$ systems (Liszt 2002).

Such models embody three primary sources of ionization and heating; soft X-rays, optical/uv photons and cosmic rays. The X-ray flux is harsh but fragile and easily attenuated by relatively small gas columns. For the reference model a "bare" intrinsic spectrum is attenuated by a nominal gas column $N_{\mathrm{w}}$ (following Wolfire et al. 1995; $N_{\mathrm{w}} \approx 10^{19} \mathrm{~cm}^{-2}$ ) whose value can be adjusted to reproduce the observed local mean density of electrons in intercloud gas (which we will also reference as pertaining to "free-space"), $\left\langle n_{\mathrm{e}}\right\rangle \approx 0.02 \mathrm{~cm}^{-3}$ (Taylor $\&$ Cordes 1993). It is a property of two-phase models that the electron density in free space varies little with the total hydrogen density $n(\mathrm{H})$ for $n(\mathrm{H})<\left\langle n_{\mathrm{e}}\right\rangle / \xi_{\mathrm{C}}$ where $\xi_{\mathrm{C}}$ is the abundance of free carbon in the gas phase.

The ionization rate of hydrogen required to ionize the intercloud gas at the observed levels is $10^{-15} \mathrm{~s}^{-1}$, about 100 times larger than the presently accepted ionization rate due to cosmic rays. This intercloud ionization rate has changed little over many years (Field et al. 1969) and is probably the bestdetermined upper limit on acceptable $\zeta_{\mathrm{H}}$ in unshielded regions. In cases with substantial intervening gas columns, the X-ray flux is propagated accordingly in our models; the spectrum hardens as the overall flux attenuates.
Table 1. Primary cosmic-ray ionization rates ${ }^{1}$ and undepleted relative abundances.

\begin{tabular}{lcc}
\hline \hline Species & $\begin{array}{c}\zeta \\
\mathrm{X}\end{array}$ & {$[\mathrm{X} / \mathrm{H}]$} \\
$\left(10^{-17} \mathrm{~s}^{-1}\right)$ & \\
\hline $\mathrm{H}$ & 1.00 & 1.00 \\
$\mathrm{D}$ & 1.00 & $1.5 \times 10^{-5}$ \\
$\mathrm{He}$ & 1.08 & $7.9 \times 10^{-2}$ \\
$\mathrm{H}_{2}$ & 2.00 & \\
$\mathrm{C}$ & 3.83 & $3.6 \times 10^{-4}$ \\
$\mathrm{~N}$ & 4.50 & $1.0 \times 10^{-4}$ \\
$\mathrm{O}$ & 5.67 & $7.5 \times 10^{-4}$ \\
\hline
\end{tabular}

${ }^{1}$ from the UMIST reaction database (http://www.rate99. co.uk)

The photoionization rate can be parametrized in terms of a scaling parameter G0, relative to a reference interstellar radiation field, but this will not be varied here. When gas parcels are modelled, the attenuating column densities are averaged appropriately over the assumed gas geometry. For $\mathrm{H}_{2}$ and $\mathrm{CO}$, appropriate shielding factors are used (Lee et al. 1996). In cool diffuse gas, the equilibrium temperature and pressure at a given density are quite sensitive to G0, and to the assumed depletion of coolants (mainly carbon).

The reference value for the primary cosmic ray ionization rate of hydrogen atoms is here taken as $\zeta_{\mathrm{H}}=10^{-17} \mathrm{~s}^{-1}$. The ionization rate of a hydrogen molecule is twice this and the total primary destruction rate of an $\mathrm{H}_{2}$ molecule due to cosmic rays, including dissociation, is $2 \times 1.08 \times \zeta_{\mathrm{H}}$. Table 1 gives the cosmic-ray ionization rates of various species scaled to the reference value of $\zeta_{\mathrm{H}}$, taken from the UMIST database (http://www.rate99.co.uk), as are all the rate constants cited here, unless otherwise noted.

\subsection{Grain neutralization}

The small grain population acquires electrons from the ambient gas by charge exchange with (neutralization of) positive ions having a superior ionization potential (typically, $6.8 \mathrm{eV}$ according to Omont 1986) and loses electrons through the photoemission processes responsible for heating. In this way, the grain heating rate is dependent on the ionization level, and both are controlled by the incident ionizing fluxes and particle density.

The neutralization rates follow from the grain charging formalism of Draine \& Sutin (1987), integrating over the grain size spectrum, assumed here, as in Bakes \& Tielens (1994) and Wolfire et al. (1995) to be an extension of the classical MRN spectrum (Mathis et al. 1977); see the last paragraph in this subsection. Weingartner \& Draine (2001a) give analytic approximations to the low-temperature charging rates (not used here) and Lepp \& Dalgarno (1988a) give a rate constant for cold gas. Grain neutralization rate constants for various ion species differ only inasmuch as their thermal velocities differ, i.e. all are proportional to the inverse square root of the atomic weight.

The abundance of small grains needed to heat the gas is about 100 times below the Solar abundance of carbon. Thus, in diffuse gas where the ionization fraction is at least equal to 
the relative abundance of free carbon (typically $40 \%$ of Solar) and grains lose electrons rapidly to photoelectric emission, the charge is carried by atomic ions and free electrons. In dense molecular gas where photoemission is nil and the equilibrium gas-phase ionization fraction falls to values below $10^{-7}$, the negative charge on grains plays a substantial role in the overall charge balance (Lepp \& Dalgarno 1988a).

Weingartner \& Draine (2001c) have recently revisited the grain spectrum and its effects on heating and cooling. They derive smaller sticking coefficients for a somewhat more copious small grain population and quote heating rates which are typically 20\%-50\% larger than those used here. By contrast, Wolfire et al. (2003) recently reduced the grain abundance and heating rate by a factor two in their reference model for local gas. In light of this conflicting guidance, we simply note that our assumptions appear to be intermediate.

\subsection{Charge exchange between oxygen and hydrogen}

The most rapid gas-phase process affecting protons in atomic gas is not recombination with electrons (or grain neutralization) but charge exchange with neutral oxygen. As originally described by Field \& Steigman (1971) in the context of a purely atomic gas, the effect of this charge exchange was to change the $\mathrm{O}^{+} / \mathrm{O}$ ratio from $10 n\left(\mathrm{H}^{+}\right) / n(\mathrm{H} \mathrm{I})$ to $\exp (-232 K / T)$ $n\left(\mathrm{H}^{+}\right) / n(\mathrm{H} \mathrm{I})$, where the exponential represents the slight difference between the ionization potentials of $\mathrm{H}$ and $\mathrm{O}$ (the latter's being larger) and the higher ionization fraction of oxygen, absent charge exchange, follows from the 1971 version of Table 1. Basically, oxygen is expected to be ionized much more rapidly than hydrogen for a given cosmic-ray flux but is forced to donate much of the ionization to hydrogen. The effect on $\mathrm{H}$ was presumed to be relatively unimportant, owing to the smallness of the $\mathrm{O} / \mathrm{H}$ ratio.

Perhaps for this reason, $\mathrm{O}+\mathrm{H}$ charge exchange was not explicitly mentioned by Wolfire et al. (1995) (it was included). However, the displacement of charge is accompanied by a slight diminution of $n(\mathrm{p})$ in our models and inclusion of charge exchange becomes important when the proton density must be determined, especially if the abundance of molecular hydrogen is considered. In even slightly denser gas having a molecular hydrogen component, $\mathrm{O}^{+}$ions interact rapidly with $\mathrm{H}_{2}$ to form $\mathrm{OH}_{3}^{+}$and this, like all molecular ions, recombines much more rapidly with electrons than does any once-ionized atom. In the absence of charge exchange with hydrogen, the equilibrium abundance of $\mathrm{O}^{+}$would be several orders of magnitude lower in gas having a molecular hydrogen fraction of even a few percent.

As noted by O'Donnell \& Watson (1974) and Barsuhn \& Walmsley (1977), maintenance of the $\mathrm{O}^{+}$fraction can actually siphon charge from hydrogen when typical diffuse cloud chemistry operates more strongly in denser but still diffuse gas. This is another effect of molecules (the first being grain neutralization) which tends to lower the proton abundance in diffuse gas. Although it is possible that a proton charge transferred to oxygen is returned to the gas after some chemical chains, this will not generally be the case. The upper limit on the

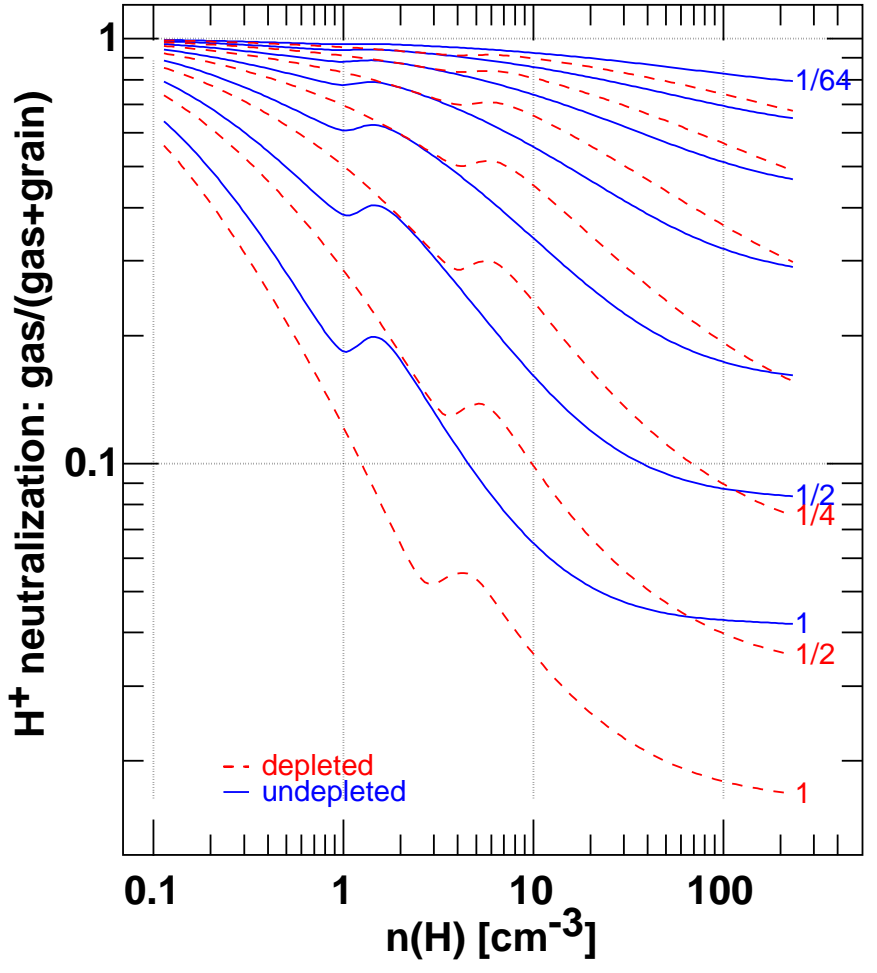

Fig. 1. The ratio of the effective gas-phase recombination rate $n(\mathrm{e}) \alpha^{(2)}$ to the total rate at which $\mathrm{H}^{+}$ions are neutralized by gas-phase recombination and grain neutralization, in two-phase equilibrium. The strength of the ion-grain interaction is artificially lowered in steps of 2 to $1 / 64$ th of the reference value. Shown are results for Solar and depleted $\left(\delta_{\mathrm{C}, \mathrm{N}, \mathrm{O}}=0.4\right)$ gas-phase abundances.

neutralization rate of protons due to oxygen charge exchange is the rate at which $\mathrm{O}^{+}$interacts with $\mathrm{H}_{2}$ to form $\mathrm{OH}^{+}$(the rate constant is $1.7 \times 10^{-9} \mathrm{~cm}^{3} \mathrm{~s}^{-1}$ ) and our models simply assume that the process is either off (in so-called atomic gas) or on at this rate whenever the abundance of $\mathrm{H}_{2}$ has been calculated. As shown below, converting $25 \%$ of a moderate density cool gas to $\mathrm{H}_{2}$ results in a decline of $30 \%-50 \%$ in $n(\mathrm{p})$.

Stancil et al. (1999) give the rate constant for the exothermic $\mathrm{O}+\mathrm{H}$ charge exchange channel as $3 \times 10^{-10} \mathrm{~cm}^{3} \mathrm{~s}^{-1}$ below $100 \mathrm{~K}$, increasing as $T^{0.23}$ for $T>100 \mathrm{~K}$. Increasing this rate has no strong direct effect on $n(\mathrm{p})$ - charge exchange already dominates - but would increase $n\left(\mathrm{O}^{+}\right)$somewhat in more strongly molecular regions, removing slightly more charge from hydrogen.

\subsection{Particulars and parameter sensitivities}

The reference model considered here assumes the small grain properties described by Bakes \& Tielens (1994); we do not adjust the grain properties except artificially to demonstrate their specific influence on the gas. Figure 1 illustrates the efficacy of $\mathrm{H}$-ion neutralization by grains, as used in the published calculations of two-phase heating and cooling Wolfire et al. (1995); Liszt $(2001,2002)$. In this figure the per-grain cross-section for neutralization is artificially varied away from the reference value (labelled 1); other aspects of the influence of small grains are not disturbed (e.g., the heating rate). The calculation is for 

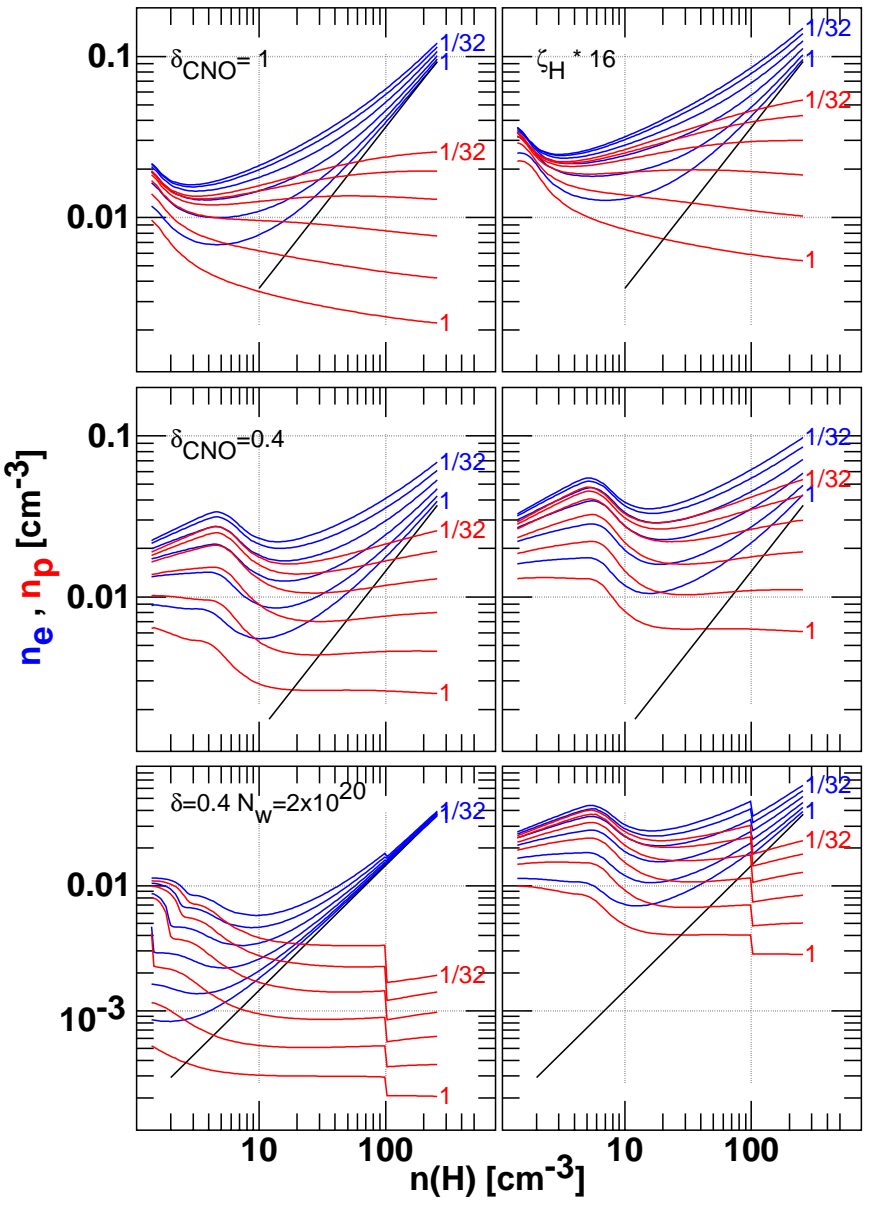

Fig. 2. Electron and proton density in two-phase equilibrium for nominal $\left(\zeta_{\mathrm{H}}=10^{-17} \mathrm{~s}^{-1}\right.$; left) and 16 times enhanced (right) cosmic-ray ionization rates under a variety of conditions. At top, for free space with Solar abundances; middle, free space with typical ISM depletions; bottom, with depletion, a substantial intervening neutral column density $\left(N_{\mathrm{w}}\right)$ shielding the soft X-ray flux and, at high density $\left(n(\mathrm{H})>100 \mathrm{~cm}^{-3}\right)$, one-fourth of the H-nuclei in $\mathrm{H}_{2}$. In each panel the strength of the grain neutralization interaction is artificially scaled from 1 times to 1/32 times the reference value (steps of 1/2). In each panel the proton density $n(\mathrm{p})$ (shown red in appropriate media) is the family of curves which lies lower at right.

free space, with and without gas-phase depletion of coolants $(\mathrm{O}, \mathrm{C})$ and charge sources $(\mathrm{C})$. Grain effects become stronger as the gas-phase ionization level declines (as could be the case in more heavily shielded regions). Clearly, the usual gas-phase recombination processes play a distinctly secondary role in cooler gas and the total rate at which protons are neutralized is much higher than has typically been assumed previously.

Shown in Fig. 2 are the electron and proton densities as functions of the total density, when the grain neutralization cross-section is altered between 1 and $1 / 32$ times the reference value, as in Fig. 1. In the top panels, no elemental gas-phase depletion is included (corresponding most nearly to the phase diagrams of Wolfire et al. 1995); below, it is taken as a typical value. In the right-hand panels, the rate of primary cosmic-ray ionization of atomic hydrogen is increased by a factor of 16 from its reference value of $1 \times 10^{-17} \mathrm{~s}^{-1}$ (all cosmic-ray rates scale in the same proportion; the rate of cosmic-ray ionization of O I, for example, is some 6 times that of H I, see Table 1). The straight line in each panel represents $n\left(\mathrm{C}^{+}\right)=3.6 \times 10^{-4} \delta_{\mathrm{C}}$ $n(\mathrm{H})$ and departures of $n(\mathrm{e})$ from it are due mostly to $n(\mathrm{p})\left(\mathrm{He}^{+}\right.$ may contribute somewhat disproportionately at lower density, owing to its high cross section for ionization by X-rays). In the lowest two panels, further complications are introduced; the gas is shielded from soft X-rays by half the hydrogen column density of a typical H I cloud (Spitzer 1968, 1978) and, for $n(\mathrm{H})>100 \mathrm{~cm}^{-3}$, a moderate fraction of the gas (one-fourth of the H-nuclei) is assumed to be molecular.

In cooler gas, neutralization of protons by small grains causes $n(\mathrm{e})$ to decrease by factors of 2-3 under reference conditions, and $n(\mathrm{p})$ decreases somewhat more. The hydrogen emission measure product $n(\mathrm{e}) n(\mathrm{p})$ typically declines by a factor 10-20 in cool material, as suggested by Fig. 1. The proton density follows $\zeta_{\mathrm{H}}$ closely only when $\mathrm{X}$-ray ionization is weak; otherwise, $n(\mathrm{p})$ increases only very sluggishly with increasing $\zeta_{\mathrm{H}}$.

\section{Ionization balance in atomic gas}

\subsection{Limits on $\zeta_{H}$ in cool gas from radio recombination lines}

Radio recombination lines (RRL) of hydrogen offer the possibility of fairly direct observation of the proton abundance. To sense the cosmic ray ionization rate, it is necessary to avoid lines of sight having any contribution from $\mathrm{H}$ II regions and to select gas which is sufficiently shielded that the X-ray ionization is weak. The electron density has a large contribution from $n(\mathrm{p})$ at all densities in unshielded regions if ion-grain neutralization is neglected, but only at low densities when it is included. In practice it has turned out to be difficult to avoid gas having a large molecular fraction, although the complications introduced in this way are never taken into account by observers.

Extant limits on the cosmic-ray ionization rate in cool atomic gas arise from (many) unsuccessful attempts to detect low-frequency (decametric) radio recombination lines (RRL) of atomic hydrogen (Shaver 1976; Payne et al. 1984, 1994). The basic analysis begins with the condition of detailed balance for hydrogen

$n(\mathrm{e}) n(\mathrm{p}) \alpha^{(2)}=\Gamma_{\mathrm{H}}(n(\mathrm{H})-n(\mathrm{p}))$

where $\Gamma_{\mathrm{H}}$ is the total ionization rate and $\alpha^{(2)}$ is the usual gas phase radiative recombination rate into the $n \geq 2$ levels, so that no ionizing photons are inadvertantly discounted. The strength of a RRL is proportional to the line of sight integral $\int n(\mathrm{e}) n(\mathrm{p}) \mathrm{d} l \equiv<n(\mathrm{e}) n(\mathrm{p})>L$ ( $L$ is some length), and to a generalized function of the properties of the host medium and the atom, accounting inter alia for non-LTE conditions among the level populations in the recombined atoms. The strength of the $21 \mathrm{~cm}$ line is assumed to be proportional to $<n(\mathrm{H} \mathrm{I})>L=<(n(\mathrm{H})-n(\mathrm{p})>L$ over the same path and to a much simpler function of temperature (assuming that the spin temperature is thermalized, a good assumption in cooler gas). 
In this case the equation of detailed balance is symbolically recast as

$\frac{\text { RRL strength }}{21 \mathrm{~cm} \text { strength }}=\frac{\Gamma_{\mathrm{H}}}{\alpha^{(2)}} F(n(\mathrm{H}), n(\mathrm{e}), T, v$, etc. $)$.

The function $F$ may have zeroes for certain combinations of density, temperature and quantum level, so the RRL to be studied must be chosen with some care. For his model of the recombination, Shaver (1976) derived limits $\zeta_{\mathrm{H}} \lesssim 2-4 \times 10^{-16} \mathrm{~s}^{-1}$ in a dozen or so $\mathrm{H}$ I features seen at low latitudes in the inner Galaxy. Many of these are fairly distant (as judged by their velocities) and most are sources of $\mathrm{CO}$ emission in galactic surveys, so they must be fairly dense; neglect of molecular processes somewhat overstates the sensitivity of the RRL measurements to $\zeta_{\mathrm{H}}$. Payne et al. (1984) set more stringent limits $\zeta_{\mathrm{H}}<0.4-1.6 \times 10^{-16} \mathrm{~s}^{-1}$ in gas toward 3C 123 which is also a well-studied molecular line source (Crutcher 1980). These limits have been considered a significant demonstration that the cosmic ray ionization rate is incapable of ionizing the intercloud gas to the observed degree (see Sect. 2.1).

In the context of the current models for ion neutralization, such limits must be revised upward to the same extent that the effective recombination rate increases (Fig. 1), which in general makes them uninteresting. Including grain neutralization reduces $n(\mathrm{p})$ by factors of 6 or so at $n(\mathrm{H}) \gtrsim 30 \mathrm{~cm}^{-3}$, and $n(\mathrm{e})$ by factors of $2-3$, which is sufficient to ensure that the soughtafter hydrogen RRL could never be observed. The RRL data really cannot be used to derive interesting limits on $\zeta_{\mathrm{H}}$, if it is accepted that grain neutralization is important.

Eventually it was recognized, largely on the basis of the behaviour of low-frequency carbon RRL (which were seen), that the function $F(n(\mathrm{H}), n(\mathrm{e}), T$, $v$, etc.) is subject to considerable uncertainty in terms of the model which is assumed for detailed balance in the recombining atoms. Payne et al. (1994) analyzed low-frequency carbon RRL and upper limits on the optical depth of the hydrogen RRL toward Cas A, and did a quite detailed heating and cooling calculation of two-phase equilibrium (excluding grain neutralization processes). Their limit on $\zeta_{\mathrm{H}}$ for one feature with an inferred density $n(\mathrm{H})=200$ $500 \mathrm{~cm}^{-3}, \zeta_{\mathrm{H}}<6 \times 10^{-17} \mathrm{~s}^{-1}$, is perhaps less interesting now than the somewhat more robust inference that $n(\mathrm{p}) / n(\mathrm{e})<0.3$. In unshielded atomic gas, such low proton fractions cannot be achieved unless grain neutralization dominates. However, the gas seen toward Cas A is dense and dark enough that it cannot be considered to be either diffuse, or atomic (Wilson et al. 1993; Liszt \& Lucas 1999). The cosmic-ray ionization rate $\zeta_{\mathrm{H}}$ is not meaningfully constrained by the Cas A data either, if grain neutralization is recognized.

\subsection{Determination of $n(e)$ via optical spectroscopy}

In their work introducing the notion of grain neutralization, Lepp et al. (1988) discussed its influence on commonlyoccuring measurements of ion ratios ( $\mathrm{Mg} \mathrm{II} / \mathrm{Mg}$ I and the like); the basic idea was that lowering such ratios with grain processes mimics the effect of higher electron and total particle densities. This in turn may help to reconcile discrepancies between densities measured from ion ratios and those gleaned

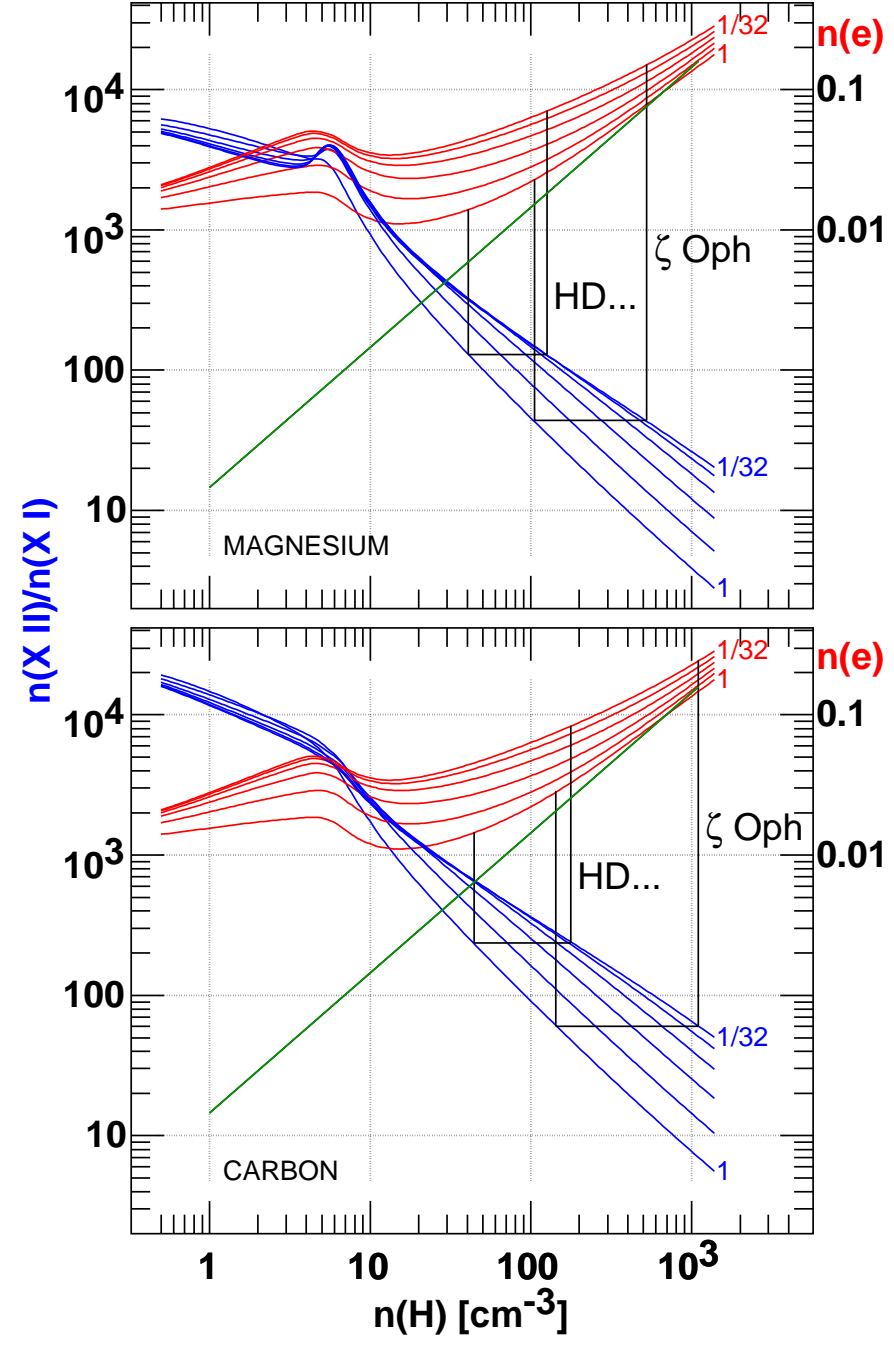

Fig. 3. Electron density (right scale; red in appropriate media) and ion stage ratios $n(\mathrm{X} \mathrm{II}) / n$ (X I) for $\mathrm{X}=$ magnesium (top) and carbon, with substantial gas-phase depletion of carbon and oxygen $\delta_{\mathrm{C}}=\delta_{\mathrm{O}}=0.4$. The ion stage ratios (shown blue in appropriate media) are the families of curves which are lower at right; families are created by scaling the grain neutralization as in Figs. 1 and 2. The ion ratios observed toward $\zeta$ Oph and HD 192639 (see Sect. 3.2) are indicated by horizontal lines and the inferred hydrogen and electron densities are given by the vertical line segments connecting the families of curves at these densities.

from study of the excitation of C I fine-structure or CO rotational levels. This mechanism has, however, only been accepted very grudgingly by observers of ion stage ratios, who are more likely to pay lip service than to calculate the effect, and most likely to sweep the matter under the rug.

Ratios of atoms in different stages of ionization depend on the total electron density, and would be sensitive to large $n(\mathrm{e})$ $n\left(\mathrm{C}^{+}\right) \approx n(\mathrm{p})$; this dependence is complicated and changed by grain neutralization, of course. If it were found that $n(\mathrm{e}) \gg$ $n\left(\mathrm{C}^{+}\right)$in sufficiently dense gas (the ionization is supported by hydrogen at low density anyway), this would suggest that $n(\mathrm{p}) / n\left(\mathrm{C}^{+}\right)$was large, and that $\zeta_{\mathrm{H}}$ was large enough to overcome whatever recombination/neutralization processes were occurring. In terms of Fig. 2, we see that this really cannot happen 
if grain neutralization occurs substantially as indicated, so high proton densities in sufficiently dense gas could be a strong contraindication of grain neutralization.

Toward $\zeta$ Oph, Savage et al. (1992) measured $N(\mathrm{Mg} \mathrm{II}) / N(\mathrm{Mg} \mathrm{I})=105$ and $N(\mathrm{Fe} \mathrm{II}) / N(\mathrm{Fe} \mathrm{I})=537$ for the denser gas at $-15 \mathrm{~km} \mathrm{~s}^{-1}$, and found $n(\mathrm{e})=0.065 \mathrm{~cm}^{-3}$ and $=0.046 \mathrm{~cm}^{-3}$ from the two ratios, respectively, based on a rather simple analysis of ionization equilibrium in an atomic gas in free space, neglecting grain neutralization and cosmic-ray ionization. For $n(\mathrm{p})=0$, these values of $n(\mathrm{e})$ would be reproduced for $n(\mathrm{H})=300-400 \mathrm{~cm}^{-3}$, $n\left(\mathrm{C}^{+}\right) / n(\mathrm{H})=1.5 \times 10^{-4}$. These densities are very nominally appropriate to the feature in question, perhaps making it appear that $n(\mathrm{p}) / n\left(\mathrm{C}^{+}\right)$is not large. However, a self-consistent application of the analysis in Spitzer (1978) shows that $n(\mathrm{p}) / n\left(\mathrm{C}^{+}\right)$would be of order unity even for $\zeta_{\mathrm{H}}=10^{-17} \mathrm{~s}^{-1}$ and $n(\mathrm{H})$ a factor of two smaller, neglecting grain neutralization. In any case, Savage et al. (1992) remarked that their values of $n(\mathrm{e})$ were an order of magnitude smaller than in the classic analysis by Morton (1975), which was based in part on the $N(\mathrm{C}$ II) $/ N(\mathrm{C} \mathrm{I})$ ratio (now measured at 60 but previously (ibid) was 30).

The close agreement in $n(\mathrm{e})$ from $\mathrm{Mg}$ and $\mathrm{Fe}$ ionization ratios was illusory, as relevant $f$-values for the $\mathrm{Mg}$ II lines were subsequently revised upward by a factor 2.4 (Fitzpatrick 1997), lowering the derived $\mathrm{Mg} \mathrm{II} / \mathrm{Mg}$ I ion ratio and requiring a substantial upward scaling of the electron density derived from it. Ion stage ratios seen toward $\zeta$ Oph are given in Fig. 3 here, which shows $n(\mathrm{X}$ II) $/ n(\mathrm{X} \mathrm{I})$ for $\mathrm{X}=$ magnesium and carbon in a free-space, two-phase heating and cooling calculation assuming a substantial depletion of gas-phase carbon and oxygen $\delta=0.4$ so that $[\mathrm{C}] /[\mathrm{H}]=1.44 \times 10^{-4}$.

In each panel of the figure the ion ratios are the families of curves which lie lower at right and higher at left; the other family in each panel is the electron density. Families of curves were created by scaling the grain neutralization rates (as in Figs. 1 and 2) from 1 to $1 / 32$ times the reference value. The shaded trapezoidal outlines in each figure illustrate where the models reproduce measured ion ratios (the horizontal base) and what electron densities are found with and (largely) without grain neutralization. The $n$ (e) values cited by Savage et al. (1992) are reproduced nearly exactly for the ion ratios they quoted.

If grain neutralization is ignored, the hydrogen (electron) densities corresponding to the $\mathrm{Mg}$ and $\mathrm{C}$ ion ratios are 530 (0.15) and $1140(0.25) \mathrm{cm}^{-3}$ respectively. At the temperature inferred for this gas from the ratio of population in the $J=1$ and $J=0$ levels of $\mathrm{H}_{2}, T=56 \mathrm{~K}$, the thermal pressure found from these ion ratios would be much higher (1020 times) than inferred from excitation of the fine-structure levels of C I (Jenkins et al. 1983) or the rotational excitation of CO (Liszt 1979; Liszt \& Lucas 1994). Including grain neutralization greatly reduces the densities, to $105(0.023)$ and 145 $(0.028) \mathrm{cm}^{-3}$, so that $n\left(\mathrm{C}^{+}\right) \gtrsim 0.66 n(\mathrm{e})$ and the $n(\mathrm{H})-T$ products derived from different analyses largely agree.

Sonnentrucker et al. (2002) present new ion stage ratios for several elements toward the low (mean) density but translucent $\left(E_{B-V}=0.64 \mathrm{mag}\right)$ line of sight to HD 192639. Their ratios for carbon and magnesium are repeated in Fig. 3; in their analysis $n(\mathrm{e}) \approx 0.1 \mathrm{~cm}^{-3}$ for either species, slightly higher than here. Along this line of sight there is a large discrepancy between the local density derived from excitation of the $\mathrm{C}$ I fine structure levels, which indicates $n(\mathrm{H})=10-20 \mathrm{~cm}^{-3}$ for $T=100 \mathrm{~K}$, and the substantially higher densities $n(\mathrm{H}) \approx 120-200 \mathrm{~cm}^{-3}$ indicated by the ion stage ratios. Sonnentrucker et al. (2002) ascribed the seemingly high electron fraction to a mixture of H I and H II gas along the line of sight but noted that their analysis might be modified by grain neutralization. Now we see that grain neuturalization does indeed remove the discrepancy.

Weingartner \& Draine (2001b) assessed existing ion stage ratios in some clouds of low inferred (from C I excitation) density $n(\mathrm{H})=10-20 \mathrm{~cm}^{-3}$ observed by Fitzpatrick \& Spitzer (1997) and found that reductions in $n(\mathrm{e})$ and the scatter among the $n(\mathrm{e})$ indicated by differing ions could be achieved, but that discrepancies between electron and particle densities derived from Ca III/Ca II and Mg II/Mg I persisted.

Welty \& Hobbs (2001) discussed a large survey of K I spectra and, in the absence of K II spectra, derived statistical properties of the cloud ensemble based on a mean value for the depletion $\delta_{\mathrm{K}}$. The median electron density $n(\mathrm{e})=0.1 \mathrm{~cm}^{-3}$ is typical of the results discussed here. These authors discussed grain neutralization at some length and concluded it was responsible for a factor three increase in $N(\mathrm{~K} \mathrm{I})$, reconciling various pressure estimates. Welty \& Hobbs (2001) noted Hobbs' long insistence that $n(\mathrm{e}) \approx n\left(\mathrm{C}^{+}\right)$for clouds seen in $\mathrm{Na} \mathrm{I}, \mathrm{K} \mathrm{I}$, etc., which we know now is only possible in diffuse gas if grain neutralization dominates.

\section{Ionization balance in diffuse gas with appreciable $\mathrm{H}_{2}$}

\subsection{Observations of $H D$}

HD does not partake of the same self-shielding which is the sine qua non of high $\mathrm{H}_{2}$-fractions. Hence the ratio $n(\mathrm{HD}) / n\left(\mathrm{H}_{2}\right)$ might be expected to be very small in diffuse gas, well below the inherent $[\mathrm{D} / \mathrm{H}]$ ratio (locally, $[\mathrm{D} / \mathrm{H}]=1.5 \times 10^{-5}$ as quoted by Moos et al. 2002). That this is not so is due to fractionation and charge exchange processes which, however uncertainly, nonetheless allow an inference of $n(\mathrm{p})$ without explicit reference to the processes by which protons are neutralized (Black \& Dalgarno 1973; Watson 1973; Jura 1974; O’Donnell \& Watson 1974; Spitzer 1978).

In a purely atomic gas ionized by cosmic rays the ionization and recombination rates of $\mathrm{H}$ and $\mathrm{D}$ atoms would be very nearly the same (the grain neutralization of $\mathrm{D}^{+}$is slower by a factor $\sqrt{2}$ ) but a strong and slightly endothermic charge exchange with protons $\mathrm{H}^{+}+\mathrm{D}+\Delta E \rightarrow \mathrm{D}^{+}+\mathrm{H}$ (rate constant $\left.k_{1}=10^{-9} \mathrm{~cm}^{3} \mathrm{~s}^{-1}, \Delta E / k=41 \mathrm{~K}\right)$ tends to force $n\left(\mathrm{D}^{+}\right) / n(\mathrm{D} \mathrm{I}) \approx$ $n\left(\mathrm{H}^{+}\right) / n(\mathrm{H} \mathrm{I}) \exp (-41 K / T)$. In the presence of $\mathrm{H}_{2}$ a rapid and relatively strongly exothermic reaction $\mathrm{D}^{+}+\mathrm{H}_{2} \rightarrow \mathrm{HD}+\mathrm{H}^{+}$ forms HD with rate constant $k_{2}=2.1 \times 10^{-9} \mathrm{~cm}^{3} \mathrm{~s}^{-11}$.

If only charge transfer and $\mathrm{H}_{2}$ fractionation neutralize $\mathrm{D}^{+} \mathrm{a}$ relatively compact expression gives $n(\mathrm{p})$ in terms of observed

\footnotetext{
1 rates in this section are taken from Table 1 of Stancil et al. (1998)
} 


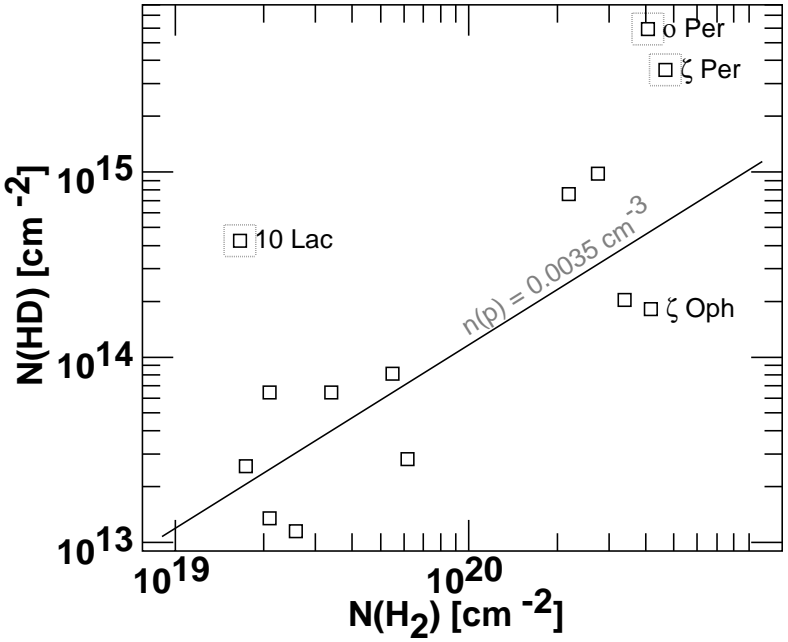

Fig. 4. $\mathrm{HD}$ and $\mathrm{H}_{2}$ column densities. $N(\mathrm{HD})$ values are from Spitzer et al. (1974) except for $o$ Per from Snow (1975), $\zeta$ Per from Snow (1977) and $\zeta$ Oph from Wright \& Morton (1979). $N\left(\mathrm{H}_{2}\right)$ values are from Savage et al. (1977). The line labelled $n(\mathrm{p})=0.0035 \mathrm{~cm}^{-3}$ is from Eq. (1), evaluated for $T=75 \mathrm{~K}$ and $\Gamma_{\mathrm{HD}}=5 \times 10^{-11} \mathrm{~s}^{-1}$ as in free space.

quantities and physical constants, with no explicit dependence on either the density or recombination rates (ibid). We have

$n(\mathrm{p})=\frac{N(\mathrm{HD}) / N\left(\mathrm{H}_{2}\right)}{[\mathrm{D} / \mathrm{H}]} \frac{\Gamma_{\mathrm{HD}}}{k_{2}}\left[1+\left(\frac{k_{2}}{k_{1}}-2\right) \frac{n\left(\mathrm{H}_{2}\right)}{n(\mathrm{H})}\right] \exp \left(\frac{41}{T}\right)$

where $n(\mathrm{H})=n(\mathrm{H} \mathrm{I})+2 n\left(\mathrm{H}_{2}\right)$ and the photodissociation rate of $\mathrm{HD} \Gamma_{\mathrm{HD}}=\Gamma_{\mathrm{H}_{2}}=5 \times 10^{-11} \mathrm{~s}^{-1}$ under typical conditions in free space (Lee et al. 1996). The derived $n(\mathrm{p})$ is nearly independent of the molecular fraction in the gas for $k_{2} / k_{1}=2.1$ and there is no explicit dependence on density (only the $\mathrm{H}_{2}$ fraction).

The available (Copernicus) observations of HD in the nearby ISM are summarized in Fig. 4; $N(\mathrm{HD})$ values are from Spitzer et al. (1974) except for $o$ Per from Snow (1975), $\zeta$ Per from Snow (1977) and $\zeta$ Oph from Wright \& Morton (1979). The $\pm 1 \sigma$ error range for $\zeta$ Per is large, 0.5 dex. With the obvious exception of the three datapoints outlined $(10 \mathrm{Lac}, o$ Per and $\zeta$ Per) the regression line fit to the data, $\log N($ HD $)=$ $-5.73+0.99 \log N\left(\mathrm{H}_{2}\right)$, has very nearly unit slope corresponding to uniform physical conditions and $N(\mathrm{HD}) / N\left(\mathrm{H}_{2}\right) \approx 1.0$ $1.2 \times 10^{-6}$. From Eq. (1) we find $n(\mathrm{p}) \approx 0.0035 \mathrm{~cm}^{-3}$ at $T=75 \mathrm{~K}$ in free space.

Thus the nominal proton densities inferred from $N(\mathrm{HD})$ are small compared to expected values of $n(\mathrm{e})$ for all but the most discrepant datapoints, and may be reproduced in slightly darker gas lacking X-ray ionization either with nominal cosmic ray ionization rates in the absence of grain neutralization, $o r$, with it, if $\zeta_{\mathrm{H}}$ is substantially enhanced (Fig. 2 at bottom). Earlier analyses attempted to infer $\zeta_{\mathrm{H}}$ from $n(\mathrm{p})$ and found an enormous range, for instance from $7 \times 10^{-18} \mathrm{~s}^{-1}$ for $\xi$ Per to $10^{-15} \mathrm{~s}^{-1}$ for $10 \mathrm{Lac}$ (O'Donnell \& Watson 1974).

\subsection{Observations of $\mathrm{H}_{3}^{+}$}

The detection of $\mathrm{H}_{3}^{+}$in diffuse gas has had some ironic twists. Chemical models employed in the original discussion of grain neutralization (Lepp \& Dalgarno 1988a; Lepp et al. 1988) were poisoned by inclusion of the then-fashionable assumption of a very small low-temperature gas-phase recombination rate for $\mathrm{H}_{3}^{+}$. This led the authors to a gross overprediction of $N\left(\mathrm{H}_{3}^{+}\right)$, which they suggested would be observable; their suggestion seems not to have been acted upon in a timely manner, thus depriving the world of a seeming corroboration of the incorrect recombination rate. Instead, the recombination rate was corrected (Amano 1988; Larsson et al. 1993; Sundstrom et al. 1994), lowering expectations for the presence of $\mathrm{H}_{3}^{+}$, and, by the time it was widely detected, this was considered surprising. With substantial fluctuations, $N\left(\mathrm{H}_{3}^{+}\right) / E_{B-V} \approx$ $8 \times 10^{14} \mathrm{~cm}^{-2} / 6$ mag (see Fig. 14 of McCall et al. 2002), or $N\left(\mathrm{H}_{3}^{+}\right) / N(\mathrm{H}) \approx 2.3 \times 10^{-8}$ for $N(\mathrm{H}) / E_{B-V}=5.8 \times 10^{21} \mathrm{~cm}^{-2}$. The clear increase in column density with line of sight extinction strongly argues that $\mathrm{H}_{3}^{+}$is widely-distributed in a relatively common cloud component of the ISM, not segregated in clouds of too-high density (which would be relatively rare).

The abundances of $\mathrm{HD}$ and $\mathrm{H}_{3}^{+}$really cannot be considered except in the broader context of a model for $\mathrm{H}_{2}$ itself (see the next subsection), but the chemistry of $\mathrm{H}_{3}^{+}$is quite simple in broad outline. Very nearly every ionization of an $\mathrm{H}_{2}$ molecule leads to formation of $\mathrm{H}_{3}^{+}$, which in diffuse gas is destroyed mainly by recombination with an electron. The rate constant for this process is taken as $\alpha_{3}=1.2 \times 10^{-7} \mathrm{~cm}^{3}(300 / T)^{0.5} \mathrm{~s}^{-1}$, which is our interpolation among experimental values which agree marginally at $300 \mathrm{~K}$ and have somewhat different temperature dependences. $\mathrm{H}_{3}^{+}$is destroyed to a much smaller extent (typically, 100 times) by reaction with $\mathrm{C}$ and $\mathrm{CO}$ (rate constant $2 \times 10^{-9} \mathrm{~cm}^{3} \mathrm{~s}^{-1}$ ) or $\mathrm{O}$ (rate constant $0.8 \times 10^{-9} \mathrm{~cm}^{3} \mathrm{~s}^{-1}$ ). The $\mathrm{CO}$ fraction in diffuse gas is quite small (cf. Fig. 8 of Liszt \& Lucas 2000), and grain neutralization at a rate $1 / \sqrt{3}$ that of $\mathrm{H}^{+}$ is also negligible in diffuse gas.

The rate equation determining the $\mathrm{H}_{3}^{+}$abundance is typically cast as $n\left(\mathrm{H}_{3}^{+}\right) n(\mathrm{e}) \alpha_{3} \approx 2 \zeta_{\mathrm{H}} n\left(\mathrm{H}_{2}\right)$ but the implicit dependences of $n\left(\mathrm{H}_{2}\right)$ and $n(\mathrm{e})$ upon $\zeta_{\mathrm{H}}$ prevent $n\left(\mathrm{H}_{3}^{+}\right)$from increasing linearly with $\zeta_{\mathrm{H}}$ for large $\zeta_{\mathrm{H}}$ as suggested by Eq. (3) of McCall et al. (2002). In our model we actually solved a small reaction network for the self-consistent determination of the abundances of the hydrogen-bearing species, including gasphase formation of $\mathrm{H}_{2}$ via $\mathrm{H}^{-}$.

\section{3. $\mathrm{H}_{3}^{+}$and $\mathrm{HD}$ in the context of $\mathrm{H}_{2}$ formation}

Figure 5 shows the run of $\mathrm{H}_{2}, \mathrm{HD}$ and $\mathrm{H}_{3}^{+}$in simple diffuse cloud models such as we have reported on in Liszt \& Lucas (2000) and Liszt (2002). A spherical clot of gas is immersed in the same conditions for which the calculations of two-phase equilibrium are performed and molecular hydrogen in the uniformly illuminated gas is allowed to self-shield. The $\mathrm{H}_{2}$ abundance is solved iteratively in 64 radial zones along with the temperature, electron density, $\mathrm{HD}$, and $\mathrm{H}_{3}^{+}$abundances etc. Represented at top in the Fig. 5 is something akin to a standard Spitzer H I cloud; at bottom is a model with four times higher number and column densities. At right, the small grains have been treated differently at the same hydrogen number and column density; at top, their neutralization activity (alone) is 
suppressed by a factor 32. At bottom they are removed from the calculation to this degree. The normalization is different for $\mathrm{H}_{3}^{+}$and $\mathrm{HD}$ because $N\left(\mathrm{H}_{2}\right)$ is generally not known along lines of sight where $N\left(\mathrm{H}_{3}^{+}\right)$was detected (only $N(\mathrm{H})$ is inferred, from the selective extinction using a standard gas-grain conversion).

Figure 5 shows that the observed abundances of HD and $\mathrm{H}_{3}^{+}$are both reproduced in the models at left, with grain neutralization and a much-elevated (factor of 10-20) cosmic ray ionization rate. In the absence of grain neutralization, either the typical $\mathrm{HD} / \mathrm{H}_{2}$ ratio can be readily reproduced with the reference value of $\zeta_{\mathrm{H}}$, or $\mathrm{H}_{3}^{+}$can be reproduced with a much (20100 times) higher value of $\zeta_{\mathrm{H}}$, at the cost of grossly overproducing HD.

Because the HD abundance is sensitive to the ionization level, it is seen to increase toward the cloud edge (the effect is only barely resolved); this would seem a natural explanation for the high $\mathrm{HD} / \mathrm{H}_{2}$ ratio toward the low $\mathrm{H}_{2}$ column density line of sight toward $10 \mathrm{Lac}$. It is also possible that HD is actually on the verge of becoming self-shielding. Observed values of $N(\mathrm{HD})$ are already larger than those of $N\left(\mathrm{H}_{2}\right)$ at the knee of the $N\left(\mathrm{H}_{2}\right)$ vs. $N(\mathrm{H})$ plot (for instance Fig. 8 of Liszt \& Lucas 2000).

The higher ionization rates seemingly demanded by a distributed $\mathrm{H}_{3}^{+}$component have been noted by McCall et al. (2002) and van der Tak $\&$ van Dishoeck (2000). The latter authors also derived values of $\zeta_{\mathrm{H}}$ from observations of $\mathrm{H}^{13} \mathrm{CO}^{+}$in the dense clouds associated with the same protostars used as background sources for the $\mathrm{H}_{3}^{+}$spectra, quoting $\zeta_{\mathrm{H}}=2.6 \pm 1.8 \times 10^{-17} \mathrm{~s}^{-1}$. Noting the discrepancy in $\zeta_{\mathrm{H}}$, they ascribed the increase of $\mathrm{H}_{3}^{+}$ with $E_{B-V}$ to intervening clouds of density $n(\mathrm{H}) \lesssim 10^{4} \mathrm{~cm}^{-3}$, in which carbon was mainly in the form of either $\mathrm{C}$ I or CO. We would take strong exception to this view, which does not account for $\mathrm{HD}$ and $\mathrm{H}_{3}^{+}$in the same gas, but the cosmic ray ionization rate in dense gas was subsequently revised by Doty et al. (2002), who quote $\zeta_{\mathrm{H}}=5.6 \times 10^{-17} \mathrm{~s}^{-1}$ with a factor three uncertainty. This does not exclude the higher values of $\zeta_{\mathrm{H}}$ preferred here. The models used by these authors do not seem to have included grain neutralization.

\section{Implications and considerations for dense gas}

\subsection{Metal depletions and the problem of sulfur chemistry in dense gas}

The Solar elemental abundances of trace metals ( $\mathrm{Mg}, \mathrm{Na}, \mathrm{Fe}$, Si etc.), $2-4 \times 10^{-5}$, while small compared to that of carbon, are much higher than the implied ionization fractions in dense dark molecular gas (see just below). Thus, even a small ionization fraction in any one of such species could dominate the overall charge balance in dark clouds.

It was noted by Oppenheimer \& Dalgarno (1974) that molecular ions will efficiently transfer charge to such metal atoms in dense gas. Because atomic ions recombine so much more slowly, in the gas (so the argument went), the overall ionization fraction is much increased in this way (if grain neutralization is neglected, as has been the case in this subject). In turn, because rather low ionization fractions are required for maintenance of high $\mathrm{H}_{3}^{+}$abundances and efficient deuteration in dense gas, chemical modellers have typically adopted what is described as a "low-metals" case, in which many metals are assumed to be strongly depleted from the gas phase $(\delta \approx 0.001)$. This includes sulfur (for example see Nillson et al. 2000), although it, unlike the other species noted just above, does not undergo charge exchange with the most abundant molecular ions $\left(\mathrm{H}_{3}^{+}, \mathrm{OH}_{3}^{+}\right.$and $\left.\mathrm{HCO}^{+}\right)$.

Such extreme sulfur depletion leads to several contradictions. Unlike, say, $\mathrm{Mg}$, $\mathrm{Na}$ or Fe, sulfur is undepleted at lower extinction (Savage \& Sembach 1996) and the patterns of gasphase depletion overall do not explain why it should be missing in denser gas. More importantly, removing so much sulfur from the gas phase makes it impossible to form commonly-observed compounds like CS, SO, etc. at the observed levels without harboring abundances of $\mathrm{OH}$ or $\mathrm{O}_{2}$ which are 100 or more times what is observed, see Turner (1995) and Nillson et al. (2000). The sulfur-bearing compounds are formed by reactions of free neutral sulfur atoms with these species and the abundance of the sulfur-bearing molecules scale linearly with the amount of free sulfur (ibid).

Although some of the discussion of Lepp \& Dalgarno (1988a) is mooted by recognition that dissociative recombination of $\mathrm{H}_{3}^{+}$is efficient, and although they did not address sulfur explicitly, these authors demonstrated that grain neutralization renders the issue of metal depletion irrelevant to the free electron abundance in dense, dark molecular gas. The charge carried by atomic ions is suppressed by grain neutralization; much of the entire negative charge contribution resides on small grains (see Sect. 2.2 here), and the free electron density varies little (less than a factor two) whether metals are depleted or present. In this case, the abundance of gas phase sulfur may safely be assumed to be large enough that models with acceptably small $\mathrm{OH}$ and $\mathrm{O}_{2}$ abundances - when they emerge - will easily produce enough of the sulfur-bearing molecules. Additionally the gas phase metals will be largely neutral, owing to grain neutralization. This acts to increase the abundances of most sulfur-bearing compounds because $\mathrm{S}^{+}$is less chemically productive. Consequences for the abundances of molecules containing other metals largely remain to be explored.

\subsection{The atomic gas fraction in dense gas}

In a dark or molecular cloud, very nearly every cosmic ray ionization results in formation of an $\mathrm{H}_{3}^{+}$ion and the ultimate appearance of $q=2-3$ hydrogen atoms (Cravens \& Dalgarno 1978). Hydrogen atoms leave the gas phase by sticking to (and forming $\mathrm{H}_{2}$ on) large grains whose density is assumed proportional to $n(\mathrm{H})=n\left(\mathrm{H}_{2}\right) / 2$. The rate constant for this process is usually denoted by $R$ and the equilibrium density of $\mathrm{H}$-atoms in a dark, fully molecular gas is $n(\mathrm{H} \mathrm{I}) \approx q \zeta_{\mathrm{H}} / R$, independent of $n\left(\mathrm{H}_{2}\right)$. Typically, $R \approx 3 \times 10^{-17} \mathrm{~cm}^{3} \mathrm{~s}^{-1}$ is inferred in diffuse gas (Jura 1974; Spitzer 1978; Gry et al. 2002), so $n(\mathrm{H} \mathrm{I}) \approx 1 \mathrm{~cm}^{-3}$, independent of $n\left(\mathrm{H}_{2}\right)$, for the reference value of $\zeta_{\mathrm{H}}$.

It has been recognized since the work of Allen \& Robinson (1976) that the fraction of atomic hydrogen inside dark clouds, while usually small, is still often much larger than expected for steady-state equilibrium between $\mathrm{H}_{2}$ formation at typical rates 


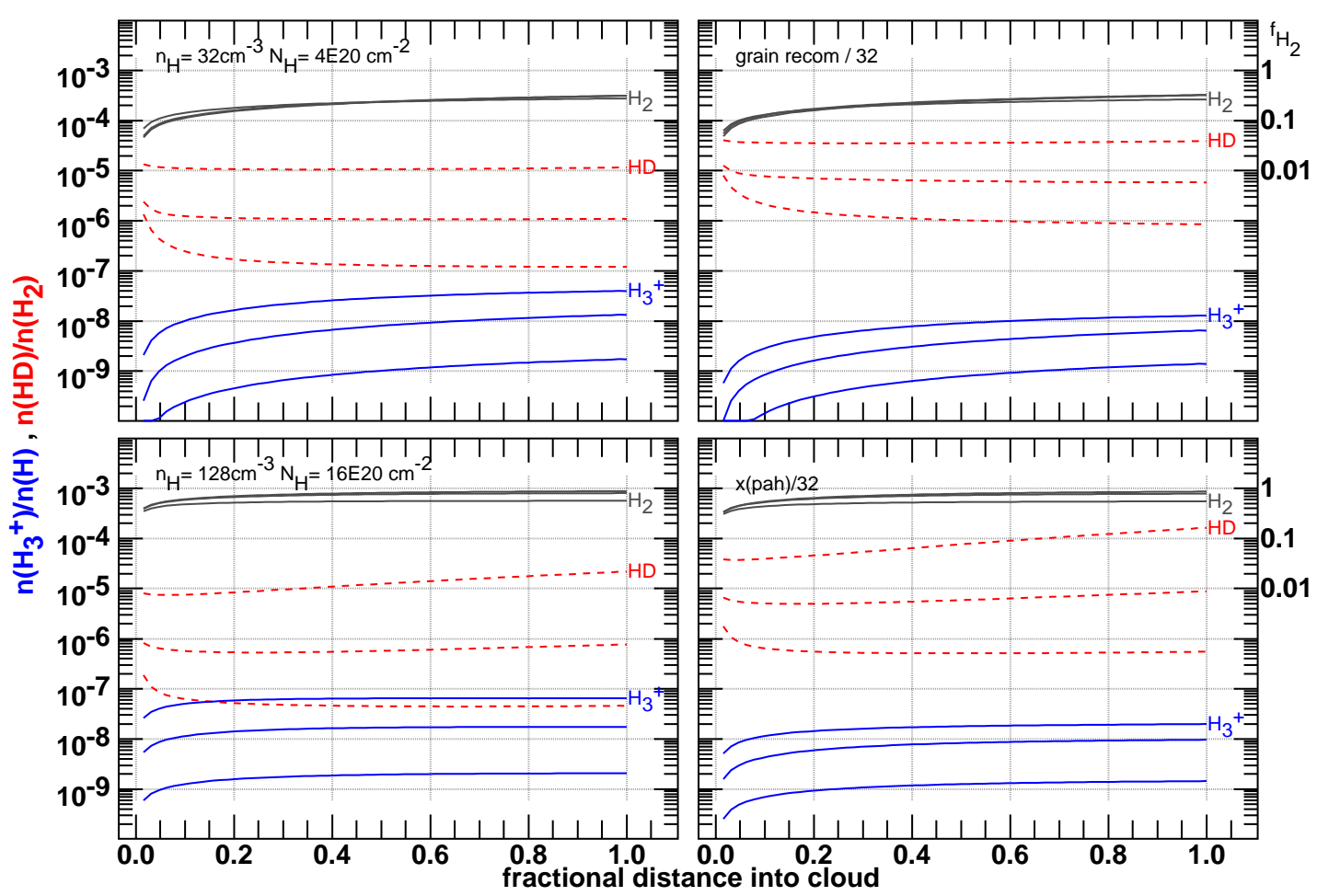

Fig. 5. Calculated $\mathrm{HD}, \mathrm{H}_{2}$ and $\mathrm{H}_{3}^{+}$fractional abundances for three values of the cosmic ray ionization rate $\zeta_{\mathrm{H}}=10^{-17} \mathrm{~s}^{-1}$, $10^{-16} \mathrm{~s}^{-1}$ and $10^{-15} \mathrm{~s}^{-1}$, for two uniformly illuminated spherical models. At top is a "standard" Spitzer (Spitzer 1968, 1978) $\mathrm{H} \mathrm{I} \mathrm{cloud} \mathrm{with} n(\mathrm{H})=32 \mathrm{~cm}^{-3}$, $N(\mathrm{H})=4 \times 10^{20} \mathrm{~cm}^{-2}$. At bottom is a model having 4 times higher number and column density. At right the effect of small grains is diluted in each model, at top by simply scaling down the grain neutralization rate, and at bottom by scaling down the quantity of small grains (and so, all associated effects). The normalizations of the relative abundances differ because $N\left(\mathrm{H}_{2}\right)$ is not known along most lines of sight with known $N\left(\mathrm{H}_{3}^{+}\right) . N(\mathrm{HD}) / N\left(\mathrm{H}_{2}\right) \approx 1.2 \times 10^{-6}$ along unexceptional lines of sight (Fig. 4) and $N\left(\mathrm{H}_{3}^{+}\right) /\left(5.8 \times 10^{21} \mathrm{~cm}^{-2} E_{B-V}\right) \approx 2.3 \times 10^{-8}$ in the survey of McCall et al. (2002).

in cold gas and a cosmic ray ionization rate $\zeta_{\mathrm{H}}=10^{-17} \mathrm{~s}^{-1}$. Observations of $\mathrm{CO}$ emission and $\mathrm{H}$ I self-absorption in lowlatitude $21 \mathrm{~cm}$ profiles by Liszt et al. $(1981,1984)$ suggested $n(\mathrm{H} \mathrm{I}) / n\left(\mathrm{H}_{2}\right) \approx 0.005$ and similarly high values have recently been cited by Willacy et al. (2002) for some local dark clouds. The latter authors invoked a process of turbulent diffusion to account for the H-fraction, while Allen \& Robinson (1976) asserted that dark clouds were not old enough for full $\mathrm{H} \mathrm{I}-\mathrm{H}_{2}$ conversion (also see Rawlings et al. 2002).

The higher values of $\zeta_{\mathrm{H}}$ suggested by this work nominally imply an enhanced atomic hydrogen fraction in fully molecular gas, in agreement with observation. However, the rate of $\mathrm{H}_{2}$ formation is really well-known only in diffuse gas, where photoprocesses dominate and grains are incompletely dressed. The rate of $\mathrm{H}_{2}$ formation in dark gas has only been interpreted in the context of the diffuse cloud data and/or relatively small $\zeta_{\mathrm{H}}$. Theoretical models, which might account for changes in the formation rate when photodesorption is nil and depletion proceeds, are largely lacking.

\subsection{The cosmic-ray heating rate in dense gas}

The standard calculation of heating in dense dark gas is that of Goldsmith (2001) who used a (cosmic-ray) heating rate $10^{-27} n\left(\mathrm{H}_{2}\right)$ erg $\mathrm{cm}^{-3} \mathrm{~s}^{-1}$. Using the mean value of the heat deposited per primary event in a weakly ionized, fullymolecular gas calculated by Cravens \& Dalgarno (1978), $\Delta Q=$ $7 \mathrm{eV}$, we find that this heating rate corresponds to $\zeta_{\mathrm{H}}=$ $4.5 \times 10^{-17} \mathrm{~s}^{-1}$, some 4.5 times our reference value; Goldsmith (2001), without attribution, assumed $\Delta Q=20 \mathrm{eV}$. Thus, increasing our primary ionization rate by a factor of 20 would provide heating which is only a factor 4.5 greater than that in Goldsmith (2001). The local cooling function varies as $T^{2.7 \pm 0.3}$ if gas-grain coupling is neglected and rather more rapidly if it is included. A substantial increase in $\zeta_{\mathrm{H}}$ (compared to our reference value) could be tolerated without inducing major changes in the temperature of dense gas; indeed, to heat molecular gas to the observed degree apparently requires a value of $\zeta_{\mathrm{H}}$ which is at least 5 times larger than our reference value.

\section{Summary}

Gas-phase atomic ions are rapidly neutralized by the same population of small grains which heats the diffuse interstellar medium. We considered the effects of such processes on various forms of hydrogen; protons in atomic gas; protons (deuterons), $\mathrm{HD}$ and $\mathrm{H}_{3}^{+}$in diffuse gas having a molecular component, and $\mathrm{H} \mathrm{I}$ in dense molecular clouds.

In atomic gas, limits on the cosmic-ray ionization rate $\zeta_{\mathrm{H}}$ (the primary rate per H-nucleus; see Table 1 ) from nondetection of low-frequency hydrogen recombination lines must 
be revised upward by such large factors that they are no longer restrictive. If grain neutralization is recognized, limits on $\zeta_{\mathrm{H}}$ from the RRL are higher than the rate needed to ionize the intercloud medium in the absence of the soft X-ray flux, as in the original theories of two-phase equilibrium. Ion stage ratios like $N(\mathrm{C}$ II $) / N(\mathrm{C} \mathrm{I}), N(\mathrm{Fe}$ II $) / N(\mathrm{Fe} \mathrm{I})$ etc. provide accurate electron densities only when grain neutralization is fully taken into account.

$\mathrm{HD}$ and the newly-discovered $\mathrm{H}_{3}^{+}$may be shown to exist in the observed amounts, even in classical cool H I clouds of moderate density, if grain neutralization proceeds and the cosmicray ionization rate has values $\zeta_{\mathrm{H}} \gtrsim 2 \times 10^{-16} \mathrm{~s}^{-1}$. Models having lower values of $\zeta_{\mathrm{H}}$ will reproduce HD if grain neutralization is neglected but fall grossly short of explaining $\mathrm{H}_{3}^{+}$.

In denser gas, a higher cosmic ray ionization rate naturally accounts for the (often) surprisingly high $\mathrm{H}$ I fractions, without invoking mechanisms such as non-equilibrium $\mathrm{H}_{2}$ formation, small cloud ages, or turbulent diffusion (all of which might still occur). More importantly, we noted that inclusion of small-grain charging in dense gas removes the need for the "low-metals" case of severe ad hoc sulfur depletion, thus allowing formation of sulfur-bearing molecules without grossly overproducing $\mathrm{OH}$ and $\mathrm{O}_{2}$.

The present work represents no more than the barest first step at including grain neutralization and its necessary consequences (like enhanced values of $\zeta_{\mathrm{H}}$ ), into our understanding of the interstellar medium.

Acknowledgements. The National Radio Astronomy Observatory is operated by Associated Universites, Inc. under a cooperative agreement with the US National Science Foundation. Comments of the referee, Dr. Mark Wolfire, are gratefully acknowledged.

\section{References}

Allen, M., \& Robinson, G. W. 1976, ApJ, 207, 745

Amano, T. 1988, ApJ, 329, L121

Bakes, E. L. O., \& Tielens, A. G. G. M. 1994, ApJ, 427, 822

Barsuhn, J., \& Walmsley, C. M. 1977, A\&A, 54, 345

Black, J. H., \& Dalgarno, A. 1973, ApJ, 184, L101

Cravens, T. E., \& Dalgarno, A. 1978, ApJ, 219, 750

Crutcher, R. M. 1980, ApJ, 239, 549

D'Hendecourt, L. B., \& Leger, A. 1987, A\&A, 180, L9

Doty, S. D., van Dishoeck, E. F., van der Tak, F. F. S., \& Boonman, A. M. S. 2002, A\&A, 389, 446

Draine, B. T., \& Sutin, B. 1987, ApJ, 320, 803

Field, G. B., Goldsmith, D. W., \& Habing, H. J. 1969, ApJ, 155, L149

Field, G. B., \& Steigman, G. 1971, ApJ, 166, 59

Fitzpatrick, E. L. 1997, ApJ, 482, L199

Fitzpatrick, E. L., \& Spitzer, L. J. 1997, ApJ, 475, 623

Geballe, T. R., McCall, B. J., Hinkle, K. H., \& Oka, T. 1999, ApJ, 510, 251

Goldsmith, P. F. 2001, ApJ, 557, 736

Gry, C., Boulanger, F., Nehmé, C., et al. 2002, A\&A, 391, 675

Jenkins, E. B., Jura, M., \& Loewenstein, M. 1983, ApJ, 270, 88

Jura, M. 1974, ApJ, 191, 375

Larsson, M., Danared, H., Mowat, J. R., et al. 1993, Phys. Rev. Lett., 70,430

Lee, H. H., Herbst, E., Pineau Des Forets, G., Roueff, E., \& Le Bourlot, J. 1996, A\&A, 311, 690

Lepp, S., \& Dalgarno, A. 1988a, ApJ, 335, 769
Lepp, S., \& Dalgarno, A. 1988b, ApJ, 324, 553

Lepp, S., Dalgarno, A., van Dishoeck, E. F., \& Black, J. H. 1988, ApJ, 329,418

Liszt, H. 2001, A\&A, 371, 698

Liszt, H. 2002, A\&A, 389, 393

Liszt, H., \& Lucas, R. 1999, A\&A, 347, 258

Liszt, H. S. 1979, ApJ, 233, L147

Liszt, H. S., Burton, W. B., \& Bania, T. M. 1981, ApJ, 246, 74

Liszt, H. S., Burton, W. B., \& Xiang, D.-L. 1984, A\&A, 140, 303

Liszt, H. S., \& Lucas, R. 1994, ApJ, 431, L131

Liszt, H. S., \& Lucas, R. 2000, A\&A, 355, 333

Mathis, J. S., Rumpl, W., \& Nordsieck, K. H. 1977, ApJ, 217, 425

McCall, B. J., Hinkle, K. H., Geballe, T. R., et al. 2002, ApJ, 567, 391

Moos, H. W., Sembach, K. R., Vidal-Madjar, A., et al. 2002, ApJS, 140,3

Morton, D. C. 1975, ApJ, 197, 85

Nillson, A., Hjalmarson, P., Bergman, P., \& Millar, T. 2000, A\&A, 358,257

O’Donnell, E., \& Watson, W. D. 1974, ApJ, 191, 89

Omont, A. 1986, A\&A, 164, 159

Oppenheimer, M., \& Dalgarno, A. 1974, ApJ, 192, 29

Payne, H. E., Anantharamaiah, K. R., \& Erickson, W. C. 1994, ApJ, 430,690

Payne, H. E., Salpeter, E. E., \& Terzian, Y. 1984, AJ, 89, 668

Rawlings, J. M. C., Hartquist, T. W., Williams, D. A., \& Falle, S. A. E. G. 2002, A\&A, 391, 681

Savage, B. D., Cardelli, J. A., \& Sofia, U. J. 1992, ApJ, 401, 706

Savage, B. D., Drake, J. F., Budich, W., \& Bohlin, R. C. 1977, ApJ, 216, 291

Savage, B. D., \& Sembach, K. R. 1996, ARA\&A, 34, 279

Shaver, P. A. 1976, Astrophysics, 49, 149

Snow, T. P. 1975, ApJ, 201, L21

Snow, T. P. 1977, Astrophysics, 216, 724

Sonnentrucker, P., Friedman, S. D., Welty, D. E., York, D. G., \& Snow, T. P. 2002, ApJ, 576, 241

Spitzer, L. 1968, Diffuse matter in space (New York: Interscience Publication, 1968)

Spitzer, L. 1978, Physical processes in the interstellar medium (New York Wiley-Interscience), 333

Spitzer, L., Cochran, W. D., \& Hirshfeld, A. 1974, ApJS, 28, 373

Stancil, P. C., Lepp, S., \& Dalgarno, A. 1998, Astrophysics, 509, 1

Stancil, P. C., Schultz, D. R., Kimura, M., et al. 1999, A\&AS, 140, 225

Sundstrom, G., Mowat, J. R., Danared, H., et al. 1994, Science, 263, 785

Taylor, J. H., \& Cordes, J. M. 1993, ApJ, 411, 674

Turner, B. E. 1995, ApJ, 455, 556

van der Tak, F. F. S., \& van Dishoeck, E. F. 2000, A\&A, 358, L79

Watson, W. D. 1973, ApJ, 182, L73

Weingartner, J. C., \& Draine, B. T. 2001a, ApJ, 548, 392

Weingartner, J. C., \& Draine, B. T. 2001b, ApJ, 563, 842

Weingartner, J. C., \& Draine, B. T. 2001c, ApJS, 134, 263

Welty, D. E., \& Hobbs, L. M. 2001, ApJS, 133, 345

Willacy, K., Langer, W. D., \& Allen, M. 2002, ApJ, 573, L119

Wilson, T. L., Mauersberger, R., Muders, D., Przewodnik, A., \& Olano, C. A. 1993, A\&A, 280, 221

Wolfire, M., McKee, C. F., Hollenbach, D., \& Tielens, A. G. G. M. 2003, ApJ, in press

Wolfire, M. G., Hollenbach, D., McKee, C. F., Tielens, A. G. G. M., \& Bakes, E. L. O. 1995, ApJ, 443, 152

Wright, E. L., \& Morton, D. C. 1979, ApJ, 227, 483 